\title{
Motivating Wittgenstein's Perspective on Mathematical Sentences as Norms ${ }^{1}$
}

\begin{abstract}
A motivation of the later Wittgenstein's perspective on mathematical sentences as norms is given for sentences belonging to axiomatic systems that are treated along the lines of the Hilbertian axiomatic method, the approach in which the axioms are used as implicit definitions of the concepts they contain. It is shown that in this approach the axioms are employed as norms in that they function as standards of what counts as using the concepts involved. This normative dimension of the mode of use of the axioms, it is argued, is inherited by the theorems derived from the axioms. Having been motivated along these lines, the Wittgensteinian perspective on mathematical language as normative may appear more plausible also to those who are not friends or experts of Wittgenstein's later philosophy of mathematics.
\end{abstract}

\section{Introduction}

Wittgenstein's later thoughts on mathematics belong to the most neglected parts of his work and have practically no influence on current debates in the philosophy of mathematics. A selection of his notes on mathematics appeared posthumously under the title Remarks on the Foundations of Mathematics $^{2}$, but it received very critical reviews by some of the leading experts in the philosophy of mathematics. ${ }^{3}$ Nevertheless, there has always been a small fraction of researchers who believe that Wittgenstein's philosophy of mathematics contains extremely important insights. ${ }^{4}$ Despite their efforts, however, Wittgenstein's thoughts on mathematics play almost no role in the more systematically oriented debates in the philosophy of mathematics today.

The aim of the present paper is to show that this neglect is unjustified. To this end, a motivation of Wittgenstein's perspective on mathematical sentences as norms is proposed which is so designed that it may appeal to those having a background in what may be called contemporary mainstream

\footnotetext{
${ }^{1}$ I would like to thank Peter Hacker, Andrea and Friedrich Harbach, Felix Mühlhölzer and two anonymous referees of Philosophia Mathematica for many valuable comments on earlier versions of this paper. I am especially grateful to Felix Mühlhölzer for motivating me to take Wittgenstein's perspective on mathematical sentences as norms seriously in the first place.

${ }^{2}$ See [Wittgenstein, 1956].

${ }^{3}$ See the review papers [Kreisel, 1958], [Bernays, 1959], and [Dummett, 1959].

${ }^{4}$ For book-length studies of aspects of Wittgenstein's (later) philosophy of mathematics see [Wright, 1980], [Baker and Hacker, 1985] (especially Chapter VII of the 2009 edition), [Shanker, 1987], [Frascolla, 1994], [Marion, 1998], and [Mühlhölzer, 2010].
} 
philosophy of mathematics. ${ }^{5}$ Before discussing the main argument, however, some brief remarks on the more general traits of Wittgenstein's thoughts on mathematics are in order to set the stage for the considerations which follow.

The later Wittgenstein's thoughts on mathematics have what one may call a positive and a negative side. On the negative side, Wittgenstein offers a profound critique of attempts to provide mathematics with a "foundation" in the sense of the logicist, intuitionist or finitist project. The foundationalist enterprise Wittgenstein is chiefly concerned with is the logicist programme of Whitehead and Russell, who tried to reconstruct mathematics as a branch of logic. His criticism, however, applies quite generally to any attempt of uncovering the nature or "essence" of mathematical objects by reductive analysis, also in a set-theoretic framework. ${ }^{6}$ In view of the anti-revisionist and descriptivist philosophical attitude championed by Wittgenstein in his later writings ${ }^{7}$ it seems more natural to interpret him as attacking (what he believes to be) most mathematicians' motivation for studying subjects such as set theory and as criticising their professed interpretations of these theories than as rejecting these theories themselves for being based on illegitimate forms of mathematical reasoning. ${ }^{8}$ Nevertheless, Wittgenstein has often been interpreted as defending various forms of constructivism and finitism, positions which reject important parts of ordinary mathematical reasoning as unintelligible. ${ }^{9}$ Wittgenstein's position, thus construed, tends to emerge as a bizarre case of philosophical presumptuousness and as, at least in part, barely sensible. In the present paper, I shall ignore what I have labelled the "negative side" of Wittgenstein's philosophy of mathematics, even though his critique of foundationalism appears to me very insightful, and focus on what I call its "positive side". ${ }^{10}$

By the "positive side" of Wittgenstein's philosophy of mathematics I mean those remarks which are not critical of any other philosophical position or programme but contain his own original, "positive", considerations on mathematics. There are several importants leitmotifs here, but the idea which is perhaps most central and ingenious is that mathematical sentences are not used descriptively, as descriptions of "mathematical facts", so to speak, but normatively, as rules of language: "[I]n mathematics we

\footnotetext{
${ }^{5}$ For an introduction see Chapters 8-10 of [Shapiro, 2001].

${ }^{6}$ The core of Wittgenstein's critique of logicist reductionism is contained in [Wittgenstein, 1956], Part III. For an extremely thorough and extensive commentary and discussion see [Mühlhölzer, 2010], for a more compact account see [Mühlhölzer, 2006].

${ }^{7}$ See [Wittgenstein, 1953], § 124 .

${ }^{8}$ For an elaborate non-revisionist reading of Wittgenstein's philosophy of mathematics, focusing on his remarks on Gödel, see [Floyd, 2001].

${ }^{9}$ See, for example, the influential reading of Wittgenstein as a "full-blooded conventionalist" proposed in [Dummett, 1959] or the Stanford Encyclopedia article on Wittgenstein's philosophy of mathematics [Rodych, 2007].

${ }^{10}$ A very useful general overview of the "negative side" of Wittgenstein's philosophy of mathematics is given in [Maddy, 1993].
} 
are convinced of grammatical propositions", Wittgenstein writes, "so the expression, the result, of our being convinced is that we accept a rule". ${ }^{11}$ Wittgenstein sees mathematics as "normative" 12 or, more figuratively, as "form[ing] a network of norms". ${ }^{13}$ In this picture of mathematics as a network of norms, the knots of the web, corresponding to the norms, represent the mathematical propositions, and the links between them are provided by the proofs.

In view of how mathematicians and philosophers of mathematics usually describe the nature of mathematical inquiry the idea that mathematical sentences are normative is a truly radical idea. As developed by Wittgenstein, it is completely at odds with any picture of the mathematician as an "explorer" or "discoverer", exploring an unchanging realm of eternal objects (the Platonist picture) or, to name another important view, exploring the most general traits of possible structures of objects (the modal structuralist picture). It is instructive to compare the Wittgensteinian perspective on mathematical language as normative to that of a logicist who, besides aiming at a reduction of mathematics to logic, subscribes to the commonplace view that logic is normative in the sense of imposing constraints on rational thought and belief. ${ }^{14}$ In particular, it is interesting to contrast Wittgenstein's view with Frege's, for the sense in which, according to Frege, logic is normative - and hence all of mathematics that can be reduced to logic - is illuminatingly different from the sense in which mathematics is normative according to Wittgenstein.

The most important difference between Frege's view and the Wittgensteinian perspective on mathematics as normative is this: For Frege, the laws of logic, to which he refers as "laws of truth", are both normative and descriptive, and the descriptive aspect is the primary one in that it constitutes their content. ${ }^{15}$ Comparing the laws of logic ("laws of truth") to the laws of nature, he claims that the latter are "general features of what happens in nature, and occurences in nature are always in accordance with them." 16 It is this descriptive sense in which Frege is chiefly interested in the laws of logic: "It is in this sense that I speak of laws of truth. Here of course it is not a matter of what happens but of what is." Whereas the laws of nature are descriptions, in general terms, of what happens in nature, the laws of logic, for Frege, describe the most general features of concepts and-both physical and non-physical —objects. ${ }^{17}$ However, Frege is far from

\footnotetext{
${ }^{11}$ See [Wittgenstein, 1956] III §26.

${ }^{12}$ See [Wittgenstein, 1956] VII $\S 61$.

${ }^{13}$ See [Wittgenstein, 1956] VII $\S 67$.

${ }^{14}$ I would like to thank an anonymous referee of Philosophia Mathematica for suggesting to me to compare the way in which mathematics is normative according to Wittgenstein to the way in which it is normative according to such a logicist.

${ }^{15}$ See [Ricketts, 1996] p. 127, [MacFarlane, 2002] p. 36.

${ }^{16}$ For this and the following citation see [Frege, 1918] p. 58.

${ }^{17}$ See [MacFarlane, 2002] p. 35, where this claim is backed up by considering, as an
} 
denying that the laws of logic are not only descriptive but also normative, for, as he claims, "[a]ny law asserting what is can be conceived as prescribing that one ought to think in conformity with it, and is thus in that sense a law of thought." 18 What is special about the laws of logic is their supreme generality in virtue of which they "prescribe universally the way in which one ought to think if one is to think at all." Logic, for Frege, is normative in that "[f]rom the laws of thought there follow prescriptions about asserting, thinking, judging, inferring" ${ }^{19}$, but this normativity is derivative insofar as it has its source in the fact that the laws of logic correctly describe the most general features of concepts and objects. ${ }^{20}$

While for Frege the laws of logic and the propositions of arithmetic impose normative constraints on what counts as "thought" because they correctly describe the most general features of concepts and objects, the normativity of mathematical sentences requires no such grounding according to Wittgenstein. Although Frege and Wittgenstein agree on the fact that logic and mathematics are normative - mathematics, for Frege, due to his logicism - , they differ on the kind of normativity in play. Whereas for Frege this normativity is derivative, mathematical sentences are first and foremost (conceptual) norms according to Wittgenstein.

The considerations that motivate Wittgenstein to think of mathematical sentences as norms for the use of mathematical concepts are very rich and complex. They are connected to his celebrated remarks on rule-following and to his highly original conception of necessity as having its roots in normativity. ${ }^{21}$ However, neither Wittgenstein's considerations on rule-following nor those on necessity and normativity are prominent topics in contemporary philosophy of mathematics. From the standpoint of modern debates on mathematics, most of Wittgenstein's thoughts look rather idiosyncratic, and it is in that sense understandable that they have so little influence.

In this paper, however, I shall argue that Wittgenstein's perspective on mathematical sentences as norms can be motivated independently of any considerations that are accessible only to friends or experts of his later philosophy. To do so, I start from something that is common ground among many of the most popular approaches in modern philosophy of mathematics, namely the Hilbertian conception of axiomatic systems in which the axioms are employed as implicit definitions of the concepts they contain. This approach has stood the test of time, both in mathematics and in the philosophy

\footnotetext{
example, a law from Grundgesetze.

${ }^{18}$ For this and the following citation see [Frege, 1893] p. xv.

${ }^{19}$ See [Frege, 1918] p. 58.

${ }^{20}$ It would be interesting to know whether adherents of Neo-Logicism, for instance Bob Hale and Crispin Wright, would consent to Frege's view on the normativity of logic. While I haven't found any evidence on this matter, there seems to be no reason to believe that their view might be significantly closer to Wittgenstein's than is Frege's.

${ }^{21}$ For an exposition and defence of this conception, see [Glock, 1996].
} 
of mathematics: It takes centre stage, for instance, in mathematical structuralism, which is perhaps the dominating paradigm in the philosophy of mathematics today. ${ }^{22}$ The claim I shall defend is that Hilbert's account of the axioms as implicit definitions leads in a very natural way to the Wittgensteinian perspective on mathematical sentences as conceptual norms - even though the overall philosophical views of mathematics defended by Hilbert and the later Wittgenstein are certainly very different.

The rest of this paper is organised as follows:

Section 2 contains a brief reminder of the axiomatic method, Hilbert's approach to the axiomatisation of mathematical theories in which the axioms are treated as implicit definitions. In Section 3 it is argued that - in accordance with Wittgenstein's idea that mathematical sentences play the role of conceptual norms - employing the axioms as implicit definitions means using them as norms governing our use of the concepts they contain. Section 4 goes on to claim that the normative aspect of how the axioms are used can be expected to carry over to any statement that has been derived from the axioms, so the mode of use of the theorems should also be normative. This completes the motivation for Wittgenstein's perspective on mathematical sentences as conceptual norms as applied to axiom systems where the axioms are treated as implicit definitions. The paper ends, in Section 5, with a brief summary and conclusion.

\section{The axioms as implicit definitions}

The notion of an axiom goes back to antiquity, but the "axiomatic method" had not been invented until the end of the 19th century. Its rise is linked to the work of Moritz Pasch and, more famously, David Hilbert, whose axiomatisation of geometry in the epoch-making Foundations of Geometry ${ }^{23}$ differs crucially from Euclid's. The defining characteristic of the Hilbertian axiomatic method, something that distinguishes this approach to axiomatic systems from earlier ones, is that the axioms are no longer interpreted as fundamental truths about mathematical (in this case geometrical) objects but as defining the very subject matter they are about. In Hilbert's account, the axioms are "definitions", but not in the more traditional sense of the term as "explicit definitions" which introduce a new term by equating an expressing containing it (the "definiendum") with another expression containing only terms assumed to be understood or already defined before (the "definiens"). The axioms, in contrast, are treated by Hilbert as "implicit definitions", which means that they are accepted as primitive truths laying

\footnotetext{
${ }^{22}$ For the relevance of Hilbert's account of axiomatics to mathematical structuralism see the introductory section of [Hellman, 2005] and Section 4.2 of the chapter on structuralism in [Shapiro, 2001].

${ }^{23}$ See [Hilbert, 1899].
} 
down connections between the concepts in terms of which they are formulated. ${ }^{24}$ To implicit definitions the question of truth or falsity according to pre-given, external criteria does not apply. The axioms, in Hilbert's account, are treated as ("internally") true when operating within the axiom system, but there is no question about their being potentially true or false according to any superordinate, external standard.

This innovative conception of axiomatics is articulated perhaps most succinctly by Hilbert himself in his correspondence with Frege. ${ }^{25}$ According to Frege, who could not make himself comfortable with Hilbert's approach to axiomatic systems and even doubted its intelligibility, the task of the axioms is to express fundamental truths. In order to accomplish this task, the axioms have to be truth-apt and must be formulated in terms of concepts the meanings of which are "assumed to be known in advance". ${ }^{26}$ In Frege's view, the meanings of mathematical concepts must therefore be fixed prior to their appearance in the axioms, otherwise the axioms "are made to carry a burden that belongs to definitions". ${ }^{27}$

Replying to Frege, Hilbert gives the following condensed formulation of his account:

In my opinion, a concept can be fixed logically only by its relations to other concepts. These relations, formulated in certain statements, I call axioms, thus arriving at the view that axioms (perhaps together with propositions assigning names to concepts) are the definitions of the concepts. I did not think up this view because I had nothing better to do, but I found myself forced into it by the requirements of strictness in logical inference and in the logical construction of a theory. I have become convinced that the more subtle parts of mathematics ... can be treated with certainty only in this way; otherwise one is going around in a circle. ([Frege, 1980] p. 51)

Hilbert apparently saw his novel conception of axiomatic systems as a major achievement and as an important step towards the enhancement of rigour and certainty in mathematical inquiry. This claim has found widespread approval among both practitioners and philosophers of mathematics. As an example, one may cite Tait who describes the "axiomatic conception" of mathematics - to axiomatise a branch of mathematics and treat the axioms

\footnotetext{
${ }^{24}$ For a more systematic characterisation of implicit definitions see [Gupta, 2009] Section 2.5. The question of what it practically means to treat an axiom as an implicit definition is the subject of Section 3.

${ }^{25}$ See [Frege, 1980]. Hilbert's contributions which are most relevant for our present purposes include a letter dated 29 December 1899 and a postcard dated 22 September 1900.

${ }^{26}$ See [Frege, 1980] p. 35

${ }^{27}$ See [Frege, 1980] p. 35.
} 
as implicit definitions of the concepts they contain - as "[t]he only conception of mathematics itself that I believe to be viable". ${ }^{28}$ The work of a mathematician employing the axiomatic method, Tait explains, can be seen as comprising two utterly distinct parts: The "dialectic" part-Tait is borrowing this terminology from Plato - which builds on our intuitions, takes into account considerations about the desired applicability of mathematics and culminates in the choice of a certain axiom system, and the genuinely mathematical part that consists in the derivation of theorems according to the rules of deductive logic. The axiomatic method enables the mathematician to design proofs of maximal rigour in the sense that every assumption that enters into the proof must have been laid down as a conceptual connection in the axioms, and only logical rules are allowed to generate theorems starting from others (including the axioms).

For the case of geometry, this is nicely explained by Shapiro:

Although spatial intuition or observation remains the source of the axioms of Euclidean geometry, in Hilbert's writing the role of intuition and observation is explicitly limited to motivation and is heuristic. Once the axioms have been formulated, intuition and observation are banished. They are not part of mathematics. ([Shapiro, 2001] p. 151)

Euclidean geometry is meant to be applicable to the study of physical space, and Euclid's choice of axioms obviously has been motivated by the properties of physical space. In Hilbert's approach, however, neither intuition nor applicability to non-mathematical systems of objects are external criteria of truth for the axioms, both can only influence our choice of an axiom system on pragmatic grounds.

Wittgenstein does not adopt (in fact never explicitly considers) the Hilbertian account of the axioms as implicit definitions in his writings, and the spirit of his considerations on mathematics is, on the whole, rather alien to that of the writings of Hilbert. Nevertheless, there is an important amount of agreement between Wittgenstein's remarks on axiomatics and the Hilbertian approach. For although, as Wittgenstein writes, an axiom may appeal to us as "self-evidently true", this is irrelevant for the role it performs in mathematical practice. Assessing the question of what it means to accept an axiom as true, he argues that "[i]t is not our finding the proposition self-evidently true, but our making the self-evidence count, that makes it into a mathematical proposition." ${ }^{29}$ This goes well with regarding the role of intuition and self-evidence as confined to motivation and heuristics, in accordance with Shapiro's characterisation of Hilbert's account of axiomatics in the passage cited before. This reading of Wittgenstein finds further support in the following passage:

\footnotetext{
${ }^{28}$ See [Tait, 2005] p. 4.

${ }^{29}$ See [Wittgenstein, 1956] IV $\S 3$.
} 
Suppose I now say: it is quite indifferent why [the axiom] is evident. It is enough that we accept it. All that is important is how we use it. ([Wittgenstein, 1956] IV §2)

When we accept a sentence as an axiom, Wittgenstein suggests, what makes it into an axiom is not that it states a self-evident truth, but the fact that we "make the self-evidence count", namely that we use it in a certain way which is characteristic of how a sentence is used if used as an axiom. This claim, however, provokes the immediate question: What are the distinctive traits of how the axioms are used if, in accordance with Hilbert's account, they are treated as implicit definitions of the concepts they contain? This question will be assessed in the following section.

\section{Implicit definitions as norms}

In this section I shall defend Wittgenstein's conception of mathematical sentences as norms with respect to the axioms as implicit definitions. To begin with, let us briefly consider the distinctive features of the linguistic usage of mathematical definitions in general - both explicit and implicit ones - and investigate what it means to employ a mathematical sentence as a definition of some term. Explicit definitions like, for example, that of "prime number" are in an important way different from implicit definitions (like the axioms à la Hilbert) in that they have two distinct "sides" connected by an equality sign, the expression to be defined occurring only on one side of the equality sign.

As an example, one may consider the (explicit) definition of "prime number", which can be given as follows:

"A prime number is a natural number which has exactly two distinct natural divisors: itself and 1." 30

With respect to explicit mathematical definitions like this one the idea that they are employed as conceptual norms should not appear far-fetched. Rather than descriptively reporting any facts about prime numbers this sentence (normatively) proposes a convention for the use of the term "prime number". This manifests itself in the fact that someone who systematically fails to correctly apply the above definition of "prime number" is less well described as having wrong opinions about (prime) numbers than as not mastering the concept of prime number at all. As the definition of "prime number" just given is aimed at being "adequate to existing usage", it qualifies as a "descriptive definition" 31 according to currently accepted terminological conventions about definitions. However, it is certainly not a

\footnotetext{
${ }^{30}$ Using the equality sign "= ${ }_{\mathrm{Df}} "$, which is characteristic of explicit definitions, this definition can be written as$$
P n={ }_{\text {Df }}(n \in \mathbb{N}) \wedge(n \neq 1) \wedge(\forall k, m \in \mathbb{N}(k \cdot m=n \rightarrow((k=1 \vee k=n) \wedge(m=1 \vee m=n)))) .
$$

${ }^{31}$ See [Gupta, 2009], Section 1.4.
} 
descriptively used sentence in the sense of being used to describe the existing usage of the term "prime number". It is much more aptly characterised as a conceptual norm in that it provides a standard of what counts as a correct deployment of the concept of prime number.

Let me conclude from this that for explicit definitions in mathematics like that of "prime number" the idea that they function as norms which govern the use of the concepts defined by means of them looks reasonable. I shall now try to generalise this result by proceeding to the axioms when treated as implicitly defining the concepts of a mathematical theory. The idea is that a class of statements may regulate the use of a number of concepts in just the same way as a single sentence may normatively govern the use of a single concept defined. Let us check whether something along these lines appears plausible for the axioms when employed as implicit definitions in the Hilbertian way. To be specific, consider, as an example, an axiom of arithmetic, say, "Every natural number has a unique successor" and imagine that it is treated as partly defining the concepts "natural number" and "successor". Is the fact that this sentence has been given the role of a definition somehow connected to a normative dimension in how it is used?

As a first step towards a positive answer we may say that the axiom licences certain conceptual connections and places a ban on others. It allows forming connections of the form "the successor of $n$ " and rules out as inadmissible sentences having the form " $(n=S k) \wedge(m=S k) \wedge(n \neq m)$ ", where " $S$ " denotes the successor function. In accordance with the considerations on definitions presented before, if the axiom is treated as implicitly defining the concepts it contains, someone apparently endorsing its negation would be ill described as a believer in falsehoods about numbers. It is more accurate to say that he does not participate in the (language) game of arithmetic at all. In a context where the axioms are treated as defining the concepts of arithmetic these concepts simply must be applied in accordance with the axioms, for otherwise the very identity of the concepts has already got lost. Anything that makes these concepts into the concepts they are is laid down in the axioms, and this is why sentences like " $(n=S k) \wedge(m=S k) \wedge(n \neq m)$ ", which are blatantly incompatible with the axioms, are immediately recognised as illegitimate moves in the language game of arithmetic. Consequently, in a rather charitable interpretation, we would interpret someone talking about "the two successors of the natural number $n$ " as proposing a conceptual framework different from that of Peano arithmetic.

Generalising these observations, we may say that whenever the axioms are used as implicit definitions to accept and endorse them is a necessary condition for using the concepts defined through them. They are thus conceptual norms in the sense that they function as standards of what counts as employing these concepts and as operating within the axiomatic framework in question. In agreement with the Wittgensteinian perspective on mathematical sentences as norms this form of normativity is primary and 
non-derivative. Whereas, according to Frege, the normative force of the laws of logic is due to the fact that they are true descriptions of the general features of concepts and objects, the axioms as implicit definitions are normative in the more direct sense that accepting them is a prerequisite for "playing the game" of using the axiomatic framework in question at all.

The normative role which, as I have argued, characterises the axioms as implicit definitions can be highlighted by stating them with an additional "Let" in front. Consider again the axiom "Every natural number has a unique successor" and think of it as - together with others - implicitly defining the concepts "natural number" and "successor". If one wants to emphasise its role as an implicit definition without having to add an additional explanation, one can do so by phrasing it as "Let every natural number have a unique successor". If "Every natural number has a unique successor" is indeed used as implicitly defining "natural number" and "successor", the two sentences should be more or less equivalent in use. With regard to the sentence starting with "Let", it is particularly striking and evident that arguing about whether or not it is true does not make much sense. The grammatical form of this sentence makes it clear that its role is that of stating a norm for the usage of the concepts "natural number" and "successor" and not that of describing anything.

During the Lectures on the Foundations of Mathematics given in Cambridge in 1939 [Wittgenstein, 1976] Wittgenstein reportedly said that "[i]n a most crude way ... the difference between an experiential proposition and a mathematical proposition which looks exactly like it ... [is that] we can always affix to the mathematical proposition a formula like 'by definition'". 32 . Affixing the formula "by definition" to a mathematical proposition is one way of designating it as a conceptual norm. Another way of doing so is to state the proposition with a "Let" in front, as I have just proposed for the axioms as implicit definitions. Whether one prefers a "Let" or a "by definition" to mark the proposition as a conceptual norm seems to be primarily a matter of taste. The only important difference, at least at this point, is that Wittgenstein regards as conceptual norms all the mathematical sentences we accept and not only the axioms, for which his perspective has now already been motivated.

Before turning our attention from the axioms to the theorems, I shall briefly address another idea that goes back to Hilbert and has strongly influenced philosophical thinking about the axioms as implicit definitions. The idea is that, if used as implicit definitions, the axioms are schematic sentences which are not about any specific objects given in advance but apply to any of a vast variety of systems of objects. In a letter to Frege, Hilbert articulates this conception as follows:

[E]very [mathematical] theory is only a scaffolding or schema of

\footnotetext{
${ }^{32}$ See [Wittgenstein, 1976] p. 111
} 
concepts together with their necessary relations to one another, and ... the basic elements can be thought of in any way one likes. If in speaking of my [geometrical] points I think of some system of things, e. g. the system: love, law, chimney-sweep ... and then assume all my axioms as relations between these things, then my propositions, e.g. Pythagoras' theorem, are also valid for these things. In other words: any theory can always be applied to infinitely many systems of basic elements. One only needs to apply a reversible one-one transformation and lay it down that the axioms shall be correspondingly the same for the transformed things. ([Frege, 1980] p. $40 \mathrm{f}$.)

For the axioms of geometry to be made true by love, law, and chimneysweep, it is necessary to (re)interpret the predicates as denoting properties of and relations between love, law, and chimney-sweep so that the axioms turn out to be true about these. Hilbert describes this as "assume all my axioms as relations between these things". There is an unlimited variety of ways of reinterpreting the predicates, and Hilbert's point seems to be that when the axioms are treated as implicit definitions all these interpretations are completely on a par and none of them is in any way privileged.

This point has found widespread approval, in particular among proponents of structuralism in the philosophy of mathematics who emphatically embrace the Hilbertian conception of the axioms as - in structuralist terminology — "defining a type of structure of mathematical interest". 33 Structuralists Hellman and Shapiro emphasise the algebraic character of the axioms in Hilbert's approach where "algebraic", according to Shapiro, means "schematic, applying to any system of objects that meets certain conditions." 34

In this context, however, it is extremely important not to misinterpret the schematicity of the axioms as a form generality, in which case the resulting idea would be that the axioms are very general sentences describing any system of objects that "meets certain conditions", to use the words of Shapiro. According to our previous considerations, the axioms are not descriptive at all, in particular not in the sense of describing a vast variety of different systems. Understanding the axioms as descriptive sentences of a very general kind, moreover, is not in agreement with Hilbert's own remarks which do not say that the axioms, if used as implicit definitions, are very general statements about an enormous variety of systems. All that Hilbert claims is that the objects the axioms are about "can be thought of in any

\footnotetext{
${ }^{33}$ See [Hellman, 2005] p. 537. Structuralism seems to be compatible with the Wittgensteinian perspective on mathematical sentences as norms motivated here if we make the important refinement that (normatively) "defining a type of structure of mathematical interest" is something that is accomplished by all mathematical sentences belonging to a mathematical theory, not only the axioms.

${ }^{34}$ See [Shapiro, 2005] p. 67.
} 
way one likes". But this just means that the axioms are not about any particular system of objects, it does not mean that the axioms, in a literal sense, are simultaneously about any system of objects we might like to think of.

Far from being a form of generality, the schematicity of the axioms emphasised by Hilbert and the structuralists can be seen as an aspect of their normativity. This normativity, as argued before, consists in the fact that the axioms, when used as implicit definitions, provide a standard of what counts as using the concepts in terms of which they are formulated. According to the Wittgensteinian perspective on the axioms developed before, the task accomplished by the axioms is a purely conceptual one insofar as applying the concepts defined in accordance with them is the one and only essential requirement for successfully operating within the axiomatic framework in question. In contrast, what conception one has of the objects the axioms are about is irrelevant for whether or not one applies the concepts defined through the axioms. Different mathematicians may well think of different types of objects and yet be participating in the same mathematical "language game". They may well communicate successfully as long as they use the terms involved in accordance with the axioms. This seems to be what Hilbert has in mind when he writes that in his account the objects the axioms are about "can be thought of in any way one likes". Conceiving of the axioms as schematic and hence algebraic - the structuralist viewand conceiving of them as conceptual norms - the view defended here - are two perspectives on the axioms as implicit definitions which differ only in emphasis. These are neither competing nor mutually exclusive views.

\section{From the axioms to the theorems}

According to Wittgenstein, as already stressed, the idea that mathematical language is used in an essentially normative way applies to mathematical sentences in general, not only the axioms. It therefore makes sense to investigate whether the account of the axioms as conceptual norms developed in the previous section can be extended to the theorems. This will be done in the present section.

There is one rather specific sense in which, according to Wittgenstein, mathematical sentences are normative. Although this sense is not of particular relevance in the present context, it has to be mentioned because it isrightly - given a strong emphasis in the existing literature on Wittgenstein's philosophy of mathematics. ${ }^{35}$ The sense in which mathematical sentences

\footnotetext{
${ }^{35}$ See, in particular [Baker and Hacker, 1985], Chapter VII ("Grammar and necessity", revised version of Chapter VI in the first edition), [Rodych, 2007], Section 3.5, and [Steiner, 2009], focusing on Wittgenstein's claim that theorems may arise as "hardened empirical regularities".
} 
are normative that I have in mind is connected to the applicability of mathematical theories outside mathematics, which, for Wittgenstein, forms an essential feature of mathematics, distinguishing it from a mere sign-game. ${ }^{36}$ The present investigation, in contrast, is concerned with the use of mathematical language within Hilbert-style axiom systems, which are part of pure mathematics.

According to Wittgenstein, mathematical language has a normative role in the application of mathematics to systems of non-mathematical objects in that mathematical sentences may function as "norms of descriptions", deciding which claims on the non-mathematical objects under consideration are meaningful and which not. The language of arithmetic, for instance, can be seen as providing norms for the counting of objects. It licences certain uses of number words in talk about empirical objects and rules out others as senseless. As an example, one may consider the sentence "Alice, Bob and Eve each ate two apples, so the three together ate seven." This sentence fails to make sense insofar as it suggests that because of the fact that Alice, Bob and Eve each ate two apples the total number of apples eaten by them equals seven, which is manifestly absurd. We can describe this aspect of the sentence - and similarly of arithmetic in general - by saying that arithmetical propositions, in this case the sentence " $3 \cdot 2 \neq 7$ ", function as norms for the counting of objects. ${ }^{37}$ However, the observation that mathematical sentences are used in a normative manner in this way is irrelevant in the context of the present investigation, where we consider the status of sentences belonging to Hilbert-style axiom systems which are part of pure mathematics. To defend the Wittgensteinian perspective with respect to such sentences in general, the account developed before with respect to the axioms has to be extended to the theorems.

At first glance, it may not appear to be difficult to draw a coherent picture in which, on the one hand, the Hilbertian conception of axiomatics is adopted and, on the other hand, the theorems are conceived of not as normative but as descriptive. The idea one is likely to have in mind is that once the concepts have been defined in the axioms there is neither a necessity nor even a possibility of fixing them any further, in any case not by the theorems. The concepts, one might think, have been given sharp boundaries in the axioms, and the theorems are subsequently used to describe the mathematical facts. The axioms, according to this perspective, do the necessary groundwork in order for the theorems to state, with maximal precision, the mathematical facts.

This line of thought, however, has something odd about it: If it were true, this would imply that there is such a thing as a derivation of descrip-

\footnotetext{
${ }^{36}$ See [Wittgenstein, 1956] V, $\S 2$.

${ }^{37}$ See Chapter VII of [Baker and Hacker, 1985], which provides an extensive discussion and forceful defence of Wittgenstein's idea that it is crucial for mathematical sentences to be used as "norms of description" in the sense discussed in the text.
} 
tively used, fact-stating sentences from purely conceptual norms. If, for a moment, we set aside the case of mathematics where we have the axioms and definitions on the one hand and the theorems on the other, the idea of such a derivation would strike most of us as quite absurd. How could this possibly work: a derivation of sentences describing some kind of external reality starting exclusively from mere conceptual norms? Returning to the case of mathematics: If all the axioms do is constraining our use of the concepts involved, how could we derive from them any truth about any realm of objects whatsoever? ${ }^{38}$

At this point, it is useful to recall from the previous section that the normative force of the axioms does not flow from the fact that they are correct descriptions of anything. Whereas for Frege the normativity of the laws of logic derives from the fact that they describe very general features of concepts and objects, the normativity of the axioms, as I have argued before, is primary and non-derivative in that to use the concepts involved in accordance with the axioms is a necessary condition for using the concepts defined through the axioms at all. An important point in the previous section was that someone who, not just by accident, endorses a statement which is blatantly incompatible with the axioms is better described as proposing a different axiomatic (i. e. conceptual) framework than as "erring" about a certain subject matter. It is dubious, however, why the incompatibility of a sentence with the axioms should have to be blatant in order for a person endorsing the sentence to be appropriately described as operating within a different conceptual framework.

Imagine a mathematician who refuses to accept a certain theorem which all others regard as validly proven and assume that it turns out that this disagreement does not arise from what both parties would regard as a mistake made by one of them. The most important remaining possible sources of disagreement are that there are different background logics in play and that the axiom systems on the basis of which the proofs are carried out are not exactly the same, even though this may have seemed so before. Whichever of these two possibilities is the case, it is arguably no less appropriate to describe the mathematician who - systematically - refuses to accept what according to all other mathematicians is a valid proof as failing to engage in the same conceptual "game" than as differing in opinion on whether what

\footnotetext{
${ }^{38}$ This remark should not be understood as promoting a form of nominalism. Wittgenstein, in particular, has no concerns about the existence of mathematical objects. For him, there is nothing wrong with calling ' $15+20=35$ ' "a statement about numbers" since it is, as he says, "what we call a statement about numbers". (See [Wittgenstein, 1976] p. 112.) The deflationary attitude towards mathematical existence expressed in this remark is much more akin to Tait's realist view (see [Tait, 2005]) than to nominalism. (See also [Gerrard, 1991], where a reading of Wittgenstein as a deflationary realist is defended.) If one wants to combine a view of mathematical sentences as norms with a non-eliminative (i. e. non-nominalist) structuralist point of view (see Fn. 33), deflationism about mathematical existence is the only choice one has.
} 
the sentence says is true.

However, nothing much hinges on whether it is really fully adequate to characterise a mathematician endorsing the negation of a theorem which all of her colleagues regard as validly proven as "failing to use the same concepts" as they do. A much more important point is that due to the theorems being mere deductive consequences of the axioms they are not in any stronger sense answerable to any kind of reality than the axioms. I have argued before that the axioms, when used as implicit definitions, are conceptual norms in a primary sense, meaning that their normativity is not derivative in the sense in which, for Frege, the normativity of the laws of logic is derivative. Moreover, the axioms are "primitive" norms in the sense that they are accepted without proof as underived statements defining the concepts they involve. The theorems, of course, are not "primitive" in that sense, simply because they are derived from other statements - namely the axioms - and acceptance of them is based on their proofs. This, however, does not bring them into closer contact to whatever kind of reality than the axioms.

In order to make these rather abstract considerations on the status of theorems in Hilbert-style axiom systems a little more vivid, it is useful to consider, as an example, the Axiom of Choice and statements which, in the context of Zermelo-Fraenkel set theory, are equivalent to it, for instance Zorn's Lemma, Zermelo's well-ordering theorem and the Hausdorff maximal principle. With regard to Zermelo-Fraenkel set theory (ZF or, if the Axiom of Choice is included, ZFC) Hilbert's account of the axioms as implicit definitions is especially natural because there exist alternative axiomatisations of set theory (like NBG set theory or Ackermann set theory) which are better described as incorporating different concepts of set than as competing accounts of what "really is true" about sets. ${ }^{39}$

Of all the statements which are equivalent to the Axioms of Choice in ZF, Zorn's Lemma, the statement that every nonempty partially ordered set in which every chain has an upper bound has at least one maximal element, is perhaps the most widely used in proofs. ${ }^{40}$ It is employed, for instance in the proof of the Hahn-Banach theorem in functional analysis, in the proof of Tychonoff's theorem in topology, and in the proof of the proposition

\footnotetext{
${ }^{39}$ For a defence of the Wittgensteinian perspective on mathematical sentences as norms with respect to a more complicated example see [Mühlhölzer, 2001], an investigation of the status of the proposition that no construction of a regular heptagon is possible within the framework of Euclidean geometry. This theorem is also discussed by Wittgenstein himself in his lectures [Wittgenstein, 1976] (in particular pp. 45-91).

${ }^{40}$ The definitions of the terms employed in the formulation of Zorn's Lemma given in the text are as follows: A partial ordering $\leq$ of a set $x$ is a reflexive, antisymmetric and transitive relation on $x$. A chain is a nonempty subset $y$ of a partially ordered set $x$ that is totally ordered with respect to $\leq(A \leq B$ or $B \leq A$ for any two elements in $y)$. An upper bound for a chain $y$ is an element $b \in x$ such that $a \leq b$ for every $a \in y$. An element $m$ of $x$ is maximal if for any $a$ such that $m \leq a$ one has $a=m$.
} 
that every vector space has a basis. There are two different ways in which Zorn's Lemma can be introduced, namely either as a theorem, derived from, for instance, the Axiom of Choice, or as a set-theoretic axiom in itself. Historically, it emerged as a statement derived from the Axiom of Choice, but there are many texts in which it is stated without proof as an "axiom of set theory" in its own right. ${ }^{41}$

The most important point about Zorn's Lemma in the context of the present investigation is that whenever it occurs in a proof it plays absolutely no role for the details of the proof whether it has been stated before as an axiom or derived as a theorem. Consider, for instance, the possibility of introducing Zorn's Lemma as an axiom and afterwards using it in the proof of the proposition that every vector space has a basis. If the considerations on the normativity of the axioms as implicit definition presented before are correct, its role will then be to licence forming the conceptual connection "maximal element of the set of linearly independent subsets of a vector space $V$ " (which turns out to designate a set of vectors forming a basis of $V$ ). The step in the proof where Zorn's Lemma is employed, however, is carried out in exactly the same way when Zorn's Lemma has been derived before from the Axiom of Choice as when it is treated as an axiom in its own right. Consequently, if the mode in which it is used when treated as an axiom can be described as (normatively) licencing a certain conceptual connection, this applies no less if one sees it as a theorem. Generalising this observation, we may conclude that for any statement which is provably equivalent to one of the axioms it makes no difference for its role in further proofs and hence for its mode of use in further mathematical activity whether it is regarded as an axiom or derived as a theorem. If, in accordance with the considerations presented before, the axioms as implicit definitions are standards of what counts as using the concepts involved, the same should be true for any theorem which, in the context of a given axiom system, is equivalent to one of the axioms.

The example of Zorn's Lemma and the Axiom of Choice in ZermeloFraenkel set theory is useful because in this case the relation between an axiom and a number of theorems is particularly easy to survey in that it is simply equivalence. There is, however, no reason to suppose that the mode of use of a theorem should depend on whether it is equivalent to one of the axioms. Without going into the details of studying cases where the logical relations between the axioms and theorems are more complicated, we may conjecture that there is no fundamental divide between the axioms and theorems as regards their mode of use, in any case not one in terms of the normative/descriptive distinction. This result confirms the expectation formulated above that the theorems, being mere deductive consequences of

\footnotetext{
${ }^{41}$ See, for example, [Grove, 1983] p. 289: "We take the point of view that the following statement, Zorn's Lemma, is an axiom of set theory". See also [Goodman, 2006] p. 168.
} 
the axioms, cannot in any stronger sense than the axioms be descriptions of anything. The motivation of Wittgenstein's perspective on mathematical sentences as conceptual norms with respect to sentences belonging to Hilbert-style axiom systems is thereby complete.

\section{Summary and conclusion}

In this paper, I have motivated Wittgenstein's idea that mathematical sentences are used as conceptual norms for sentences belonging to axiomatic systems that are treated along the lines of the Hilbertian axiomatic method. In a first step, it was shown that the axioms in Hilbert-style axiom systems are employed as conceptual norms in the sense that they function as standards of what counts as employment of the concepts defined through them. In a second step, it was argued that the same normative mode of use that applies to the axioms characterises the theorems which are derived from them. While in virtue of being derived from the axioms the theorems are not in the same sense "primitive norms" as the axioms, they are not in any stronger sense than the axioms answerable to any kind of reality, so they are no more descriptive than these. Since the normativity of the axioms is primary in that it does not arise from the fact that the axioms are correct descriptions of anything, the same should hold for the theorems.

Hilbert's and Wittgenstein's overall philosophical views on mathematics are, of course, very different. However, an important element of Wittgenstein's thoughts on mathematics - his perspective on mathematical sentences as conceptual norms - is arguably not in conflict with an important element of Hilbert's approach to axiomatics - his conception of the axioms as implicit definitions. Here I have tried to motivate the first from the latter by outlining a route from the (nowadays) common account of the axioms championed by Hilbert to the more exotic perspective on mathematical language as normative developed by Wittgenstein.

\section{References}

Baker, G. and Hacker, P. M. S., Wittgenstein: Rules, Grammar and Necessity Volume 2 of an Analytical Commentary on the Philosophical Investigations, 2nd edition (2009), Oxford: Wiley-Blackwell (1985).

Bernays, P., Comments on Ludwig Wittgenstein's 'Remarks on the Foundations of Mathematics', Ratio 2 (1959): 1-22.

Dummett, M., Wittgenstein's Philosophy of Mathematics, The Philosophical Review 68 (1959): 324-348.

Floyd, J., Prose versus Proof: Wittgenstein on Gödel, Tarski, and Truth, Philosophia Mathematica (3) 9 (2001): 280-307. 
Frascolla, P., Wittgenstein's Philosophy of Mathematics, London and New York: Routledge (1994).

Frege, G., Grundgesetze der Arithmetik, begriffsschriftlich abgeleitet, Jena: Pohle, 2 vols. (1893) and (1903), partially transl. by Furth, M. as The Basic Laws of Arithmetic: Exposition of the System, Berkeley: University of California Press (1964).

Frege, G., Philosophical and Mathematical Correspondence, edited by McGuinness, B., translated by Kaal, H., Oxford: Blackwell (1980).

Frege, G., Der Gedanke: Eine logische Untersuchung, Beiträge zur Philosophie der Deutschen Idealismus 1:58-77 (1918), transl. as "Thoughts" by Geach, P. and Stoothoff, R. H., in McGuinness, B. (ed.) Collected Papers on Mathematics, Logic, and Philosophy, Oxford: Blackwell (1984).

Gerrard, S., Wittgenstein's Philosophies of Mathematics, Synthese, 87 (1991): 125142.

Glock, H.-J., Necessity and Normativity, in: The Cambridge Companion to Wittgenstein, edited by Sluga, H. and Stern, D. G., Cambridge: Cambridge University Press (1996): 198-225.

Goodman, F. M., Algebra: Abstract and Concrete-ed. 2.5, Iowa City: Semisimple Press (2006).

Grove, L. C., Algebra, New York: Academic Press (1983).

Gupta, A., Definitions, The Stanford Encyclopedia of Philosophy (Spring 2009 Edition), E. N. Zalta (ed.), URL = http://plato.stanford.edu/archives/spr2009/entries/definitions/.

Hellman, G., Structuralism, in: The Oxford Handbook of the Philosophy of Mathematics and Logic, edited by Shapiro, S., Oxford: Oxford University Press (2005): 536-562.

Hilbert, D., Grundlagen der Geometrie, Leipzig: Teubner (1899), Foundations of Geometry, trans. by Townsend, E., La Salle, Illinois: Open Court (1959).

Kreisel, G., Wittgenstein's Remarks on the Foundations of Mathematics, British Journal for the Philosophy of Science IX, 34 (1958): 135-158.

MacFarlane, J., Frege, Kant, and the Logic in Logicism, The Philosophical Review, 111(1) (2002): 25-65.

Maddy, P., Wittgenstein's Anti-Philosophy of Mathematics, in: Wittgenstein's Philosophy of Mathematics, edited by Puhl, K., Vienna: Verlag Hölder-PichlerTempsky (1993): 52-72.

Marion, M., Wittgenstein, Finitism, and the Foundations of Mathematics, Oxford: Oxford University Press (1998).

Mühlhölzer, F., Wittgenstein and the Regular Heptagon, Grazer Philosophische Studien 62 (2001): 215-247. 
Mühlhölzer, F., A Mathematical Proof Must Be Surveyable-What Wittgenstein Meant by this and what it Implies, Grazer Philosophische Studien 71 (2006): $57-86$.

Mühlhölzer, F., Braucht die Mathematik eine Grundlegung? Ein Kommentar des Teils III von Wittgensteins 'Bemerkungen über die Grundlagen der Mathematik', Frankfurt a. M.: Vittorio Klostermann (2010).

Ricketts, T., Logic and Truth in Frege, Proceedings of the Aritotelian Society 70 (1996): 121-140.

Rodych, V., Wittgenstein's Philosophy of Mathematics, The Stanford Encyclopedia of Philosophy (Fall 2008 Edition), E. N. Zalta (ed.), $\mathrm{URL}=$ http://plato.stanford.edu/archives/fall2008/entries/wittgensteinmathematics/.

Shanker, S. G., Wittgenstein and the Turning Point in the Philosophy of Mathematics, London: Croom Helm (1987).

Shapiro, S., Thinking about Mathematics: The Philosophy of Mathematics, Oxford: Oxford University Press (2001).

Shapiro, S., Categories, Structures, and the Frege-Hilbert Controversy: The Status of Meta-mathematics, Philosophia Mathematica (3) 13 (2005): 61-77.

Steiner, M., Empirical Regularities in Wittgenstein's Philosophy of Mathematics, Philosophia Mathematica (3) 17 (2009): 1-34.

Tait, W., The Provenance of Pure Reason. Essays on the Philosophy of Mathematics and its History, New York and Oxford: Oxford University Press (2005).

Wittgenstein, L., Philosophical Investigations, 4th edition (2009), trans. G. E. M. Anscombe, P. M. S. Hacker and J. Schulte, Oxford: Blackwell (1953).

Wittgenstein, L., Remarks on the Foundations of Mathematics, revised edition (1978), trans. G. E. M. Anscombe, Oxford: Blackwell (1956).

Wittgenstein, L., Lectures on the Foundations of Mathematics, Cambridge, 1939, edited by Cora Diamond, Hassocks: Harvester Press (1976).

Wright, C., Wittgenstein on the Foundations of Mathematics, London: Duckworth (1980). 